\title{
Is Bariatric Surgery a Prophylaxis for Pelvic Floor Disorders?
}

\author{
Andrzej Pomian ${ }^{1}$ - Wojciech Majkusiak ${ }^{1}$ - Wojciech Lisik ${ }^{2}$ - Paweł Tomasik ${ }^{1}$ - Edyta Horosz ${ }^{1}$ - Aneta Zwierzchowska ${ }^{1}$. \\ Jacek Kociszewski ${ }^{3} \cdot$ Ewa Barcz $^{1}$ (i)
}

Published online: 18 December 2017

(C) The Author(s) 2017. This article is an open access publication

\begin{abstract}
Introduction Obesity is one of the well-documented risk factors of pelvic floor disorders (PFDs). The PFDs include urinary and fecal incontinence (UI, FI) and pelvic organ prolapse (POP). Surgery-induced weight loss improves different kinds of incontinence as well as POP symptoms. However, there is a lack of evidence how bariatric surgery influences pelvic floor anatomy and function in women without previous PFDs and whether it may be concerned as PFD prophylaxis tool.

Materials and Methods The present analysis is a prospective, non-randomized case-control study from January 2014 to September 2017. Participants underwent pelvic floor ultrasound examination with bladder neck position estimation at rest, during levator ani tension, and at Valsalva maneuver before surgery and 12-18 months after. Pelvic organ prolapse quantification $(\mathrm{POPQ})>2$ stage and PFD complaints were the exclusion criteria.

Results Fifty-nine patients underwent bariatric surgery ( 57 sleeve gastrectomy and 2 gastric bypass). Mean BMI decreased from $43.8 \pm 5.9$ to $29 \pm 4.6 \mathrm{~kg} / \mathrm{m}^{2}$ after surgery $(p<0.001)$. Statistically significant higher position of the bladder neck at rest, during tension, and at Valsalva maneuver $(p<0.05)$ was shown after surgery. We did not demonstrate differences in bladder neck mobility and bladder neck elevation at tension after weight loss.

Conclusions Bariatric surgery is associated with a betterment of bladder neck position at rest, tension, and Valsalva maneuver in women without PFDs. We postulate that bariatric surgery may be a tool for PFD prevention. It does not improve levator ani function and does not limit bladder neck mobility, which implicates that it has no influence on preexisting pelvic dysfunction.
\end{abstract}

Keywords Bariatric surgery $\cdot$ Pelvic organ prolapse $\cdot$ Pelvic floor disorders $\cdot$ Urethral mobility $\cdot$ Female incontinence $\cdot$ Obesity

\section{Introduction}

Obesity is defined as a body mass index (BMI) greater than $30 \mathrm{~kg} / \mathrm{m}^{2}$. It is a worldwide public health problem as it has a negative impact on the individual's well-being and is a risk for many chronic diseases (metabolic syndrome, musculoskeletal disorders, and certain types of cancer). The prevalence of obesity has doubled within the last three decades and currently almost $15 \%$ of the world population is obese [1].

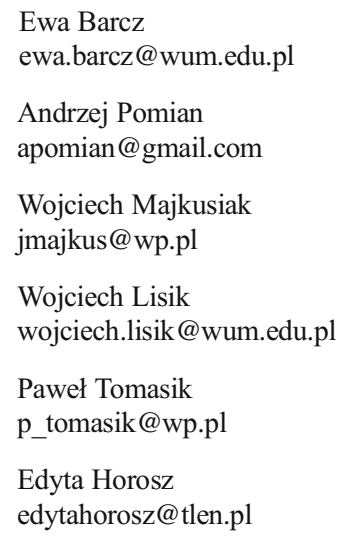

Aneta Zwierzchowska teksanskamasakra@o2.pl

Jacek Kociszewski

kociszewski@evk-haspe.de

1 1st Department of Obstetrics and Gynecology, Medical University of Warsaw, Pl. Starynkiewicza 1/3, 02-015 Warsaw, Poland

2 Department of General and Transplantation Surgery, Medical University of Warsaw, Warsaw, Poland

3 Evangelisches Krankenhaus Hagen-Haspe, Hagen, Germany 
Pelvic floor disorders (PFDs) encompass a broad spectrum of health problems, including different types of urinary incontinence (UI), pelvic organ prolapse (POP), fecal incontinence (FI), and defecatory and sexual dysfunctions. PFDs influence medical, emotional, social, and economic issues of women all over the world. It is estimated that different conditions connected with pelvic floor disorders concern approximately $30 \%$ of adult women population worldwide with increased incidence in elderly and obese population [2].

There are many risk factors for developing pelvic floor disorders including vaginal and instrumental deliveries, age, race, family history, and last but not least, overweight and obesity [3]. Women with obesity are at much higher risk as compared to normal-weight individuals for developing different types of incontinence and POP. It is estimated that over $50 \%$ of women with a BMI greater than $35 \mathrm{~kg} / \mathrm{m}^{2}$ report a PFD, compared with approximately $30 \%$ of women with a normal body mass index [4].

There are strong evidences that reducing weight improves urinary incontinence. It was shown that after bariatric surgery, there were significant improvements in voiding status assessed by voiding questionnaires [5], as well as in objective tests such as pad test [6]. Additionally, it was confirmed that weight loss after surgery improves various lower urinary tract symptoms such as stress urinary incontinence, urge incontinence, and dysuria, as well as quality of life in the above aspect [7]. It was also shown on the basis of patients' questionnaires that bariatric surgery improves different symptoms related to pelvic floor disorders connected with POP (prolapse, lower urinary tract, colorectal symptoms, and sexual dysfunctions) $[8,9]$.

Most current studies on the influence of bariatric surgery on PFDs focus on subjective improvement of symptoms, basing on different kinds of questionnaires and rarely rate objective signs of pelvic floor anatomy and function. Moreover, authors usually concentrate on already existing symptoms of PFDs. Till now, there is no data if and how weight loss may influence pelvic floor anatomy and/or function in women without PFDs and whether it may serve as a prophylaxis for future possible failure.

The urethro-vesical junction (bladder neck) is a point that corresponds to point Aa in POPQ (pelvic organ prolapse quantification scale) on the anterior vaginal wall $3 \mathrm{~cm}$ from the vaginal vestibule. Lowering of its position is connected with anterior vaginal wall descent as well as with higher risk of urinary incontinence. Bladder neck hypermobility (the difference between bladder neck positions at rest vs during Valsalva maneuver) is connected with higher incidence of stress UI [10] whereas the levator ani injury may be the cause of bladder neck descent and future pelvic floor dysfunctions and prolapse [11]. Elevation of the bladder neck during tension is realized by contraction of the most important muscle of the pelvic floor-levator ani. The measurement of the above parameter may serve as an indirect indicator of its function. The aim of the present study was to evaluate whether BMI reduction after bariatric surgery influences bladder neck position as well as its mobility and levator ani function in obese women without previous history of pelvic floor dysfunction.

\section{Material and Methods}

The present analysis is a prospective non-randomized singlecenter case-control study, approved by local ethic committee before initiation.

Fifty-nine adult obese women (BMI > 35) who were scheduled for bariatric surgery were included in the trial. The inclusion criteria were obesity, no PFD symptoms, and POPQ examination $<2$ within all compartments. All patients underwent pelvic floor ultrasound examination with evaluation of three parameters: bladder neck position at rest, during levator ani tension, and during maximum Valsalva maneuver. The control examination took place minimum 1 year after bariatric surgery.

Bladder neck position was measured in a standardized manner, with the patient on the gynecological chair in a semi sitting position with the bladder filled to $200-400 \mathrm{ml}$ (the association between the bladder filling and bladder neck position in the volume range of $200-400 \mathrm{ml}$ was not statistically significant). Three diameters of the bladder was measured in order to estimate the bladder volume at the beginning of the examination. The probe (a 3.6-8.3-MHz vaginal transducer with a beam angle of $160^{\circ}$ ) was placed in the vaginal introitus at the level of the external urethral orifice. With the probe in this position, the bladder neck (BN), urethra $(\mathrm{U})$, and pubic symphysis (PS) with the interpubic disc were visualized in the median sagittal plane, according to Interdisciplinary S2k Guideline: Sonography in Urogynecology [12].

Bladder neck position at rest was measured as the shortest distance between the point of urethral-bladder junction and the horizontal line running through the lower edge of symphysis pubis and was shown in millimeters. Accordingly, bladder neck position was measured in maximal descent point during Valsalva maneuver (Fig. 1) and in maximal elevation point at contracting levator ani muscle (Fig. 2).

Bladder neck mobility was defined as the difference between its position at rest and during Valsalva maneuver and was shown in millimeters. Bladder neck elevation during contraction was shown as the difference between bladder neck position at rest and during levator ani muscle tension.

Descriptive statistical analysis and statistical tests were performed using the R version 3.4.0 (by the R Foundation for Statistical Computing). Normality was tested using Lilliefors and Shapiro-Wilk $W$ tests. We associated the degree and type of non-adherence using the Wilcoxon signed-rank test and multivariate variance analysis (MANOVA) and multiple 
Fig. 1 Measurement of bladder neck position at rest: the shortest distance between the point of urethral-bladder junction and the horizontal line running through the lower edge of symphysis pubis (marked with white line) and bladder neck position measurement in maximal descent point during Valsalva maneuver (marked with the red line). SP symphysis pubis, $\mathrm{U}$ urethra, $\mathrm{BN}$ bladder neck

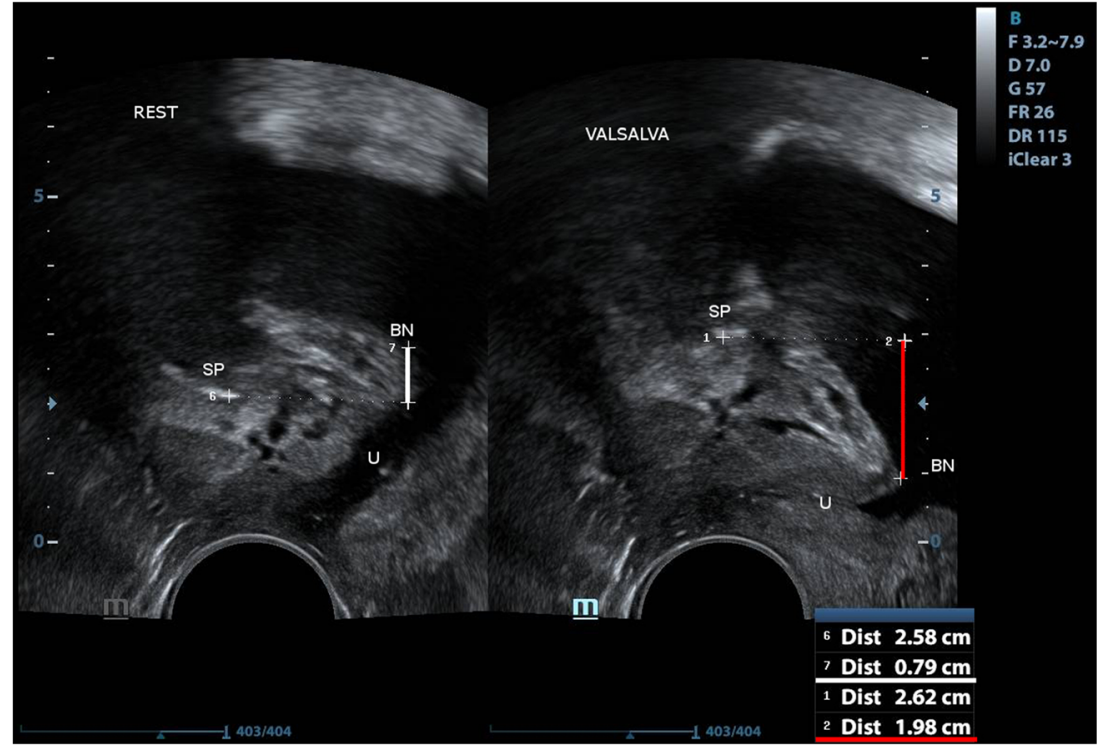

regression for multivariable analysis. We established a significance level of $p<0.05$.

\section{Results}

Fifty-nine women without PFDs were enrolled in the study. Demographic features of the study group are shown in Table 1.

In all the examined cases, significant weight loss was observed after bariatric surgery $\left(43.7 \pm 5.8 \mathrm{vs} 29 \pm 4.6 \mathrm{~kg} / \mathrm{m}^{2}\right)$.

We showed statistically significant elevation of the bladder neck position at rest in patients who underwent bariatric surgery $(p=0.004) 15.2 \pm 5.4$ vs $17.6 \pm 4.0 \mathrm{~mm}$. Significantly higher position of the bladder neck at tension $(p=0.004)$
$20.3 \pm 5.7$ vs $22.9 \pm 5.1 \mathrm{~mm}$ as well as during Valsalva maneuver $(p=0.03$ ) $3.0 \pm 7.9$ vs $5.1 \pm 7.7 \mathrm{~mm}$ was observed after weight loss (Fig. 3).

Age, parity, mode of delivery, and hormonal status did not influence the observed changes in bladder neck position at rest, tension, and Valsalva maneuver in multivariable analysis.

The calculation of absolute value of change of the bladder neck position at rest vs at levator ani tension showed no differences in muscle function following weight lost after bariatric surgery $5.1 \pm 3.8$ vs $5.4 \pm 3.8$ (n/s, $p=0.94$ ) (Fig. 4).

Similarly, we did not show changes in bladder neck mobility after bariatric surgery shown in absolute values as the difference of bladder neck position at rest vs Valsalva maneuver $12.2 \pm 6.7$ vs $12.4 \pm 6.6(\mathrm{n} / \mathrm{s}, p=0.34)($ Fig. 5$)$.
Fig. 2 Measurement of bladder neck position at rest: the shortest distance between the point of urethral-bladder junction and the horizontal line running through the lower edge of symphysis pubis (marked with the white line) and bladder neck position measurement in maximal elevation point at contracting levator ani muscle (marked with the red line). SP symphysis pubis, $\mathrm{U}$ urethra, BN bladder neck

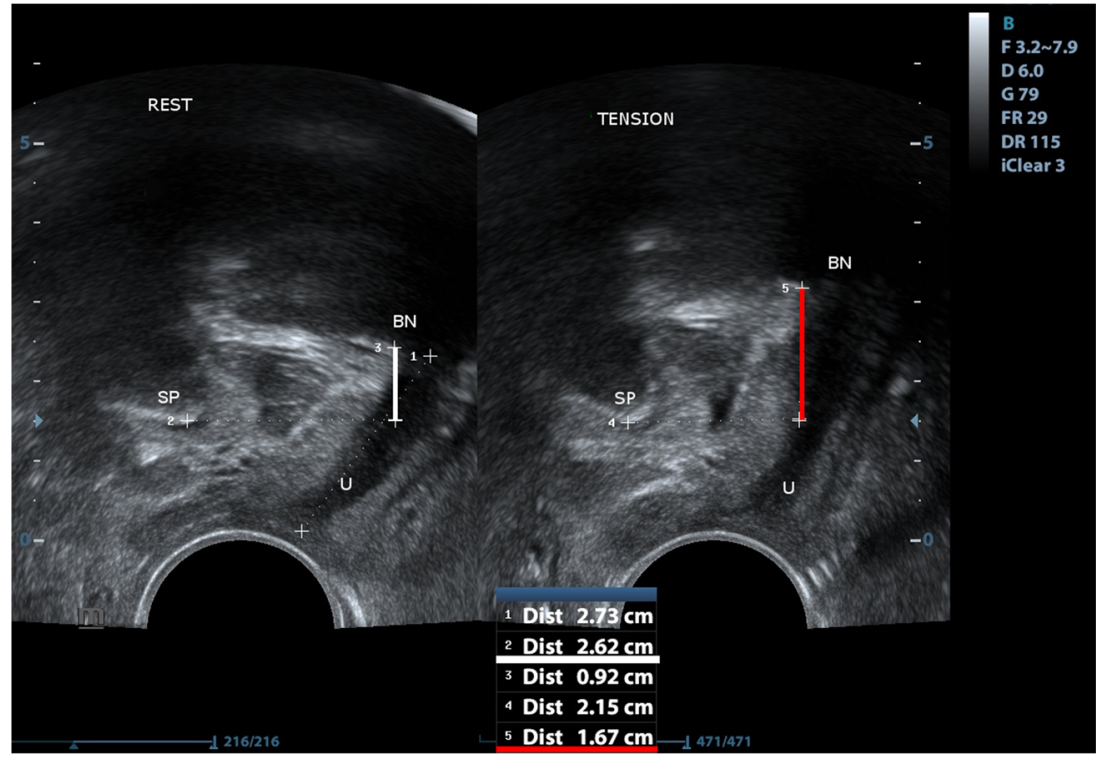


Table 1 Demographic features of examined group

\begin{tabular}{llc}
\hline & Before surgery & After surgery \\
\hline Age & $42.2 \pm 11.8$ & $43.5 \pm 11.5$ \\
BMI & $43.7 \pm 5.8$ & $29 \pm 4.6$ \\
\%EWL & $81.3 \pm 22.9 \%$ & \\
\%total body weight loss & $33.9 \pm 8.7 \%$ & \\
Parity & $1.5 \pm 1.3$ & \\
Nulliparas & $32.2 \%$ & \\
Menopause & $32.2 \%$ & \\
Surgery type & 57 patients-SG & \\
& 2 patients-RYGB \\
\hline
\end{tabular}

$B M I$ body mass index, \%EWL \% excess weight loss, $S G$ sleeve gastrectomy, $R Y G B$ Roux-en-Y gastric bypass

\section{Discussion}

Obesity is considered as one of the most important risk factors of pelvic floor disorders. It is suggested that increased intraabdominal pressure causes weakening of pelvic floor muscles and destruction of the fascia leading to pelvic organ prolapse and incontinence [13]. Moreover, obesity is associated with impairment of the quality of life (QOL) as far as pelvic floor symptoms are concerned [14].

Among pelvic floor disorders' risk factors, only several are modifiable. There has been ongoing discussion to what extent delivery mode or elective cesarean section may prevent PFDs in high-risk subjects [15]. There are also strong evidences that pelvic floor muscle training improves POP and incontinence [16]. As far as the influence of weight loss on PFDs is concerned, it has been shown that bariatric surgery improves QOL and self-reported prolapse symptoms [17]. However, till now, there have been no reports concerning the influence of bariatric surgery on the anatomical and functional features of pelvic floor in patients without PFD. The questions seem to be of a great importance as possible protective effect of surgery- induced weight loss might become one of the important issues in patients' counseling.

In the current study, for the first time, it was shown that BMI reduction results in the betterment of bladder neck position in patients without clinically manifested $\mathrm{POP}$ - one of the objective and measurable features of the pelvic floor anatomy. At the same time, we showed higher position of the bladder neck during tension of levator ani as well as during Valsalva maneuver. Most of current studies draw the conclusion about PFD improvement on the basis of patients' questionnaires showing positive subjective results of bariatric surgery on the pelvic floor anatomy and function and they seem to be in agreement with our observations, which may be an objective explanation of such subjective improvement [18].

On the basis of the above observations, we postulate that weight loss may be a prophylaxis tool in the prevention of pelvic floor disorders probably in the mechanism of lowering of the intra-abdominal pressure. The above results might be an important argument when counseling patients before bariatric surgery.

On the other hand, we did not show any improvement in levator ani function measured as absolute value of the difference between bladder neck position at rest vs at levator ani tension. It may suggest that weight loss itself does not restore muscle function and other medical options should be considered.

The present work has also shown that bladder neck mobility measured as the difference of the bladder neck position at rest vs Valsalva maneuver was not restricted after bariatric surgery. It suggests that bladder neck hypermobility and descent that are often connected with urinary incontinence and POP do not change, and therefore, all abnormalities connected with pelvic floor injuries or weakening do not restore together with BMI normalization. It stays in agreement with observations showing no improvement of already existing POP symptoms after bariatric surgery [19].

In the current literature, there are strong evidences that weight loss results in at least partial resolution of incontinence symptoms. Nevertheless, it has been suggested that urinary
Fig. 3 Bladder neck position at rest, tension, and during Valsalva maneuver before and after bariatric surgery with marked values density and 0.95 confidence interval $(n=59)$

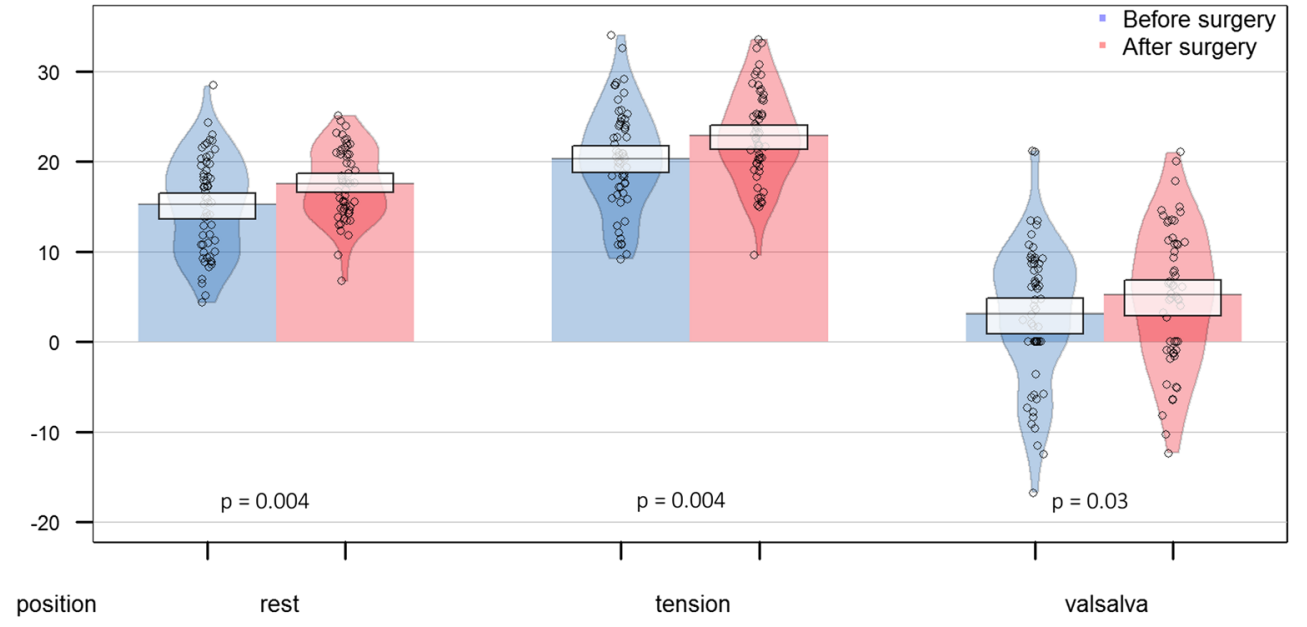




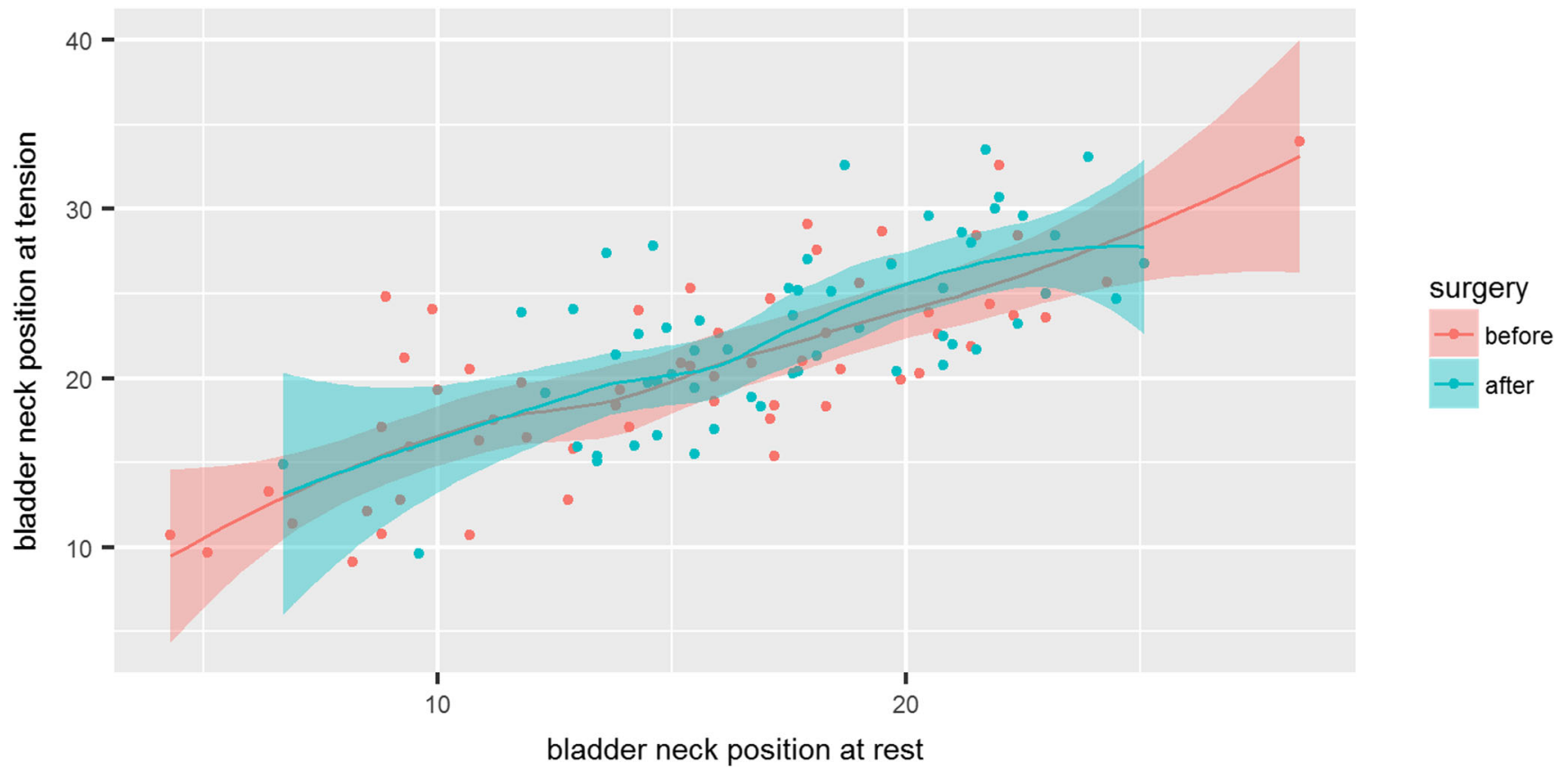

Fig. 4 Scatterplot of bladder neck position at rest vs at tension before and after surgery with marked 0.95 confidence interval. The degree of inclination of the trend line corresponds to the levator ani function before (red) and after surgery (green) and does not differ significantly

incontinence in obese women is dependent rather on higher intra-vesical pressure than urethral hypermobility [20]. The above observation may explain incontinence improvement after bariatric surgery despite lack of restoration of bladder neck mobility that has been demonstrated in the current study.

All the above observations suggest that weight normalization after bariatric surgery improves anatomical features of the pelvic floor but at the same time does not change preexisting functional conditions. Therefore, it should be emphasized in the process of patients' counseling that weight loss should result in betterment of pelvic floor anatomy as long as there is no serious impairment, but it will not restore muscle functions and preexisting fascia injuries resulted from, i.e., labors and obesity. Therefore, it should be taken into consideration that the sooner surgery-induced weight loss is obtained, the greater the chance for preserving pelvic floor wellness.

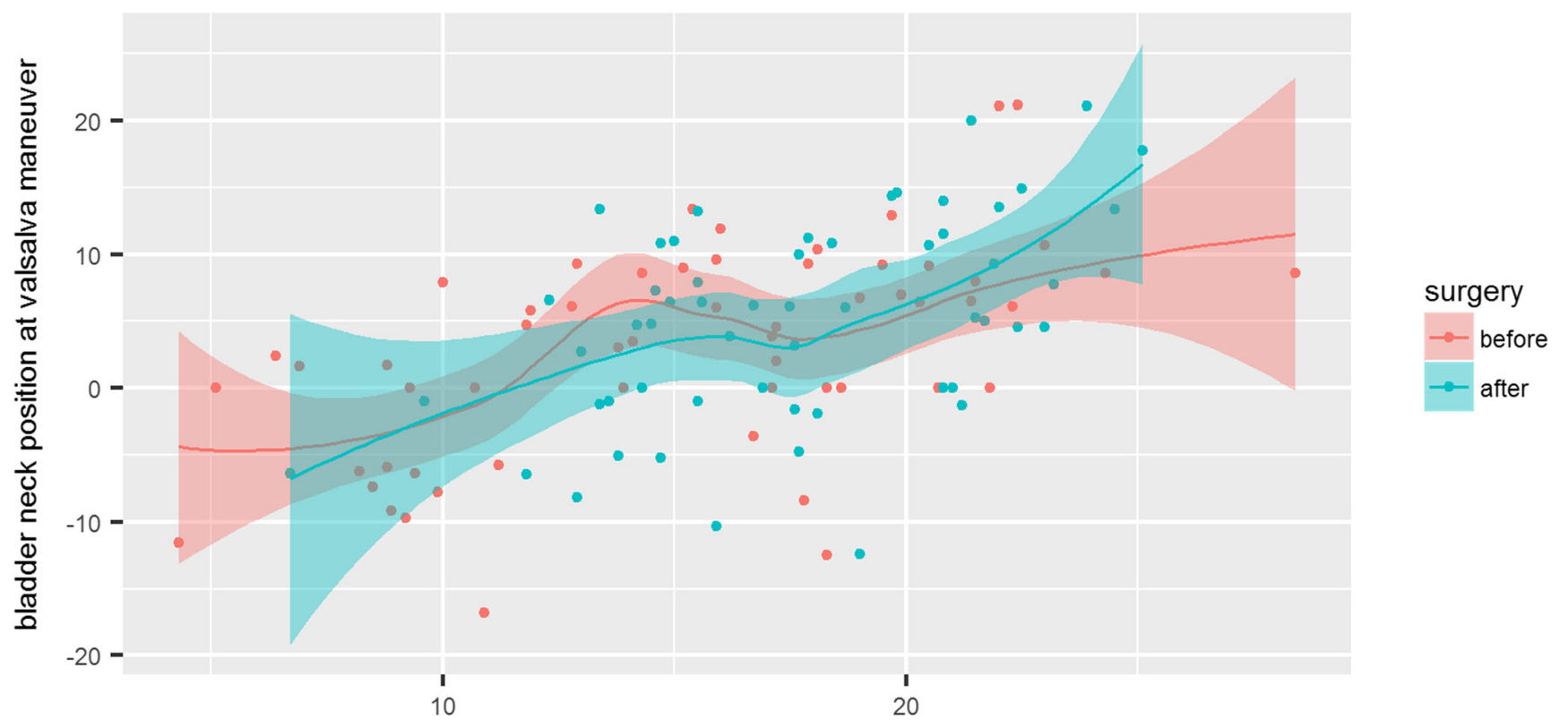

bladder neck position at rest

Fig. 5 Scatterplot of bladder neck position at rest vs at tension before and after surgery with marked 0.95 confidence interval. The degree of inclination of the trend line corresponds to the urethral mobility before (red) and after surgery (green) and does not differ significantly 


\section{Conclusions}

Bariatric surgery is associated with a betterment of bladder neck position at rest, at tension, and during Valsalva maneuver in women without PFDs. On the basis of the above observations, we postulate that bariatric surgery may be a tool for PFD prevention resulting in bladder neck elevation probably through the reduction of intra-abdominal pressure. At the same time, it does not improve levator ani function and does not limit bladder neck mobility having no influence on preexisting pelvic floor functional features.

Compliance with Ethical Standards The protocol for the research project was approved by the ethics committee of Medical University of Warsaw and it conforms to the Declaration of Helsinki. Informed consent was obtained from all individual participants included in the study.

Conflict of Interest The authors declare that they have no conflict of interest.

Open Access This article is distributed under the terms of the Creative Commons Attribution 4.0 International License (http:// creativecommons.org/licenses/by/4.0/), which permits unrestricted use, distribution, and reproduction in any medium, provided you give appropriate credit to the original author(s) and the source, provide a link to the Creative Commons license, and indicate if changes were made.

\section{References}

1. Stevens GA, Singh GM, Lu Y, et al. National, regional, and global trends in adult overweight and obesity prevalences. Popul Health Metrics. 2012;10(1):22. https://doi.org/10.1186/1478-7954-10-22.

2. Zeleke BM, Bell RJ, Billah B, et al. Symptomatic pelvic floor disorders in community-dwelling older Australian women. Maturitas. 2016;85:34-41. https://doi.org/10.1016/j.maturitas. 2015.12.012.

3. Barber MD. Pelvic organ prolapse. BMJ. 2016;354:i3853.

4. de Sam LS, Nardos R, Caughey AB. Obesity and pelvic floor dysfunction: battling the bulge. Obstet Gynecol Surv. 2016;71(2):114 25 .

5. Scozzari G, Rebecchi F, Giaccone C, et al. Bariatric surgery improves urinary incontinence but not anorectal function in obese women. Obes Surg. 2013;23(7):931-8. https://doi.org/10.1007/ s11695-013-0880-8

6. O'Boyle CJ, O'Sullivan OE, Shabana H, et al. The effect of bariatric surgery on urinary incontinence in women. Obes Surg. 2016;26(7):1471-8. https://doi.org/10.1007/s11695-015-1969-z.
7. Ait Said K, Leroux Y, Menahem B, et al. Effect of bariatric surgery on urinary and fecal incontinence: prospective analysis with 1-year follow-up. Surg Obes Relat Dis. 2017;13(2):305-12. https://doi. org/10.1016/j.soard.2016.08.019.

8. Romero-Talamas H, Unger CA, Aminian A, et al. Comprehensive evaluation of the effect of bariatric surgery on pelvic floor disorders. Surg Obes Relat Dis. 2016;12(1):138-43. https://doi.org/10.1016/j. soard.2015.08.499.

9. Knepfler T, Valero E, Triki E, et al. Bariatric surgery improves female pelvic floor disorders. J Visc Surg. 2016;153(2):95-9. https://doi.org/10.1016/j.jviscsurg.2015.11.011.

10. Naranjo-Ortiz C, Shek KL, Martin AJ, et al. What is normal bladder neck anatomy? Int Urogynecol J. 2016;27(6):945-50. https://doi. org/10.1007/s00192-015-2916-1.

11. Volloyhaug I, van Gruting I, van Delft K, et al. Is bladder neck and urethral mobility associated with urinary incontinence and mode of delivery 4 years after childbirth? Neurourol Urodyn. 2017;36(5): 1403-10. https://doi.org/10.1002/nau.23123.

12. Tunn R, Albrich S, Beilecke K, et al. Interdisciplinary S2k guideline: sonography in urogynecology: short version-AWMF registry number: 015/055. Geburtshilfe Frauenheilkd. 2014;74(12):1093-8. https://doi.org/10.1055/s-0034-1383044.

13. Lee UJ, Kerkhof MH, van Leijsen SA, et al. Obesity and pelvic organ prolapse. Curr Opin Urol. 2017;27(5):428-34. https://doi. org/10.1097/MOU.0000000000000428.

14. Chen CC, Gatmaitan P, Koepp S, et al. Obesity is associated with increased prevalence and severity of pelvic floor disorders in women considering bariatric surgery. Surg Obes Relat Dis. 2009;5(4): 411-5. https://doi.org/10.1016/j.soard.2008.10.006.

15. Howard D, Makhlouf M. Can pelvic floor dysfunction after vaginal birth be prevented? Int Urogynecol J. 2016;27(12):1811-5. https:// doi.org/10.1007/s00192-016-3117-2.

16. Hagen S, Glazener C, McClurg D, et al. Pelvic floor muscle training for secondary prevention of pelvic organ prolapse (PREVPROL): a multicentre randomised controlled trial. Lancet. 2017;389(10067): 393-402. https://doi.org/10.1016/S0140-6736(16)32109-2.

17. Cuicchi D, Lombardi R, Cariani S, et al. Clinical and instrumental evaluation of pelvic floor disorders before and after bariatric surgery in obese women. Surg Obes Relat Dis. 2013;9(1):69-75. https://doi.org/10.1016/j.soard.2011.08.013.

18. Leshem A, Shimonov M, Amir H, et al. Effects of bariatric surgery on female pelvic floor disorders. Urology. 2017;105:42-7. https:// doi.org/10.1016/j.urology.2017.03.003.

19. Lian W, Zheng Y, Huang H, et al. Effects of bariatric surgery on pelvic floor disorders in obese women: a meta-analysis. Arch Gynecol Obstet. 2017;296(2):181-9. https://doi.org/10.1007/ s00404-017-4415-8.

20. Swenson CW, Kolenic GE, Trowbridge ER, Berger MB, LewickyGaupp C, Margulies RU, et al. Obesity and stress urinary incontinence in women: compromised continence mechanism or excess bladder pressure during cough? Int Urogynecol J. 2017. 